\title{
Assessment of Drug Release Kinetics and Quality of Naproxen Generic Tablets in Bangladesh
}

\author{
Madhabi Lata Shuma ${ }^{1}$, Amrin Jahan ${ }^{1}$, and Shimul Halder2 ${ }^{2 *}$ \\ ${ }^{1}$ Department of Pharmacy, Stamford University Bangladesh, Siddeswari Dhaka, Bangladesh. \\ ${ }^{2}$ Department of Pharmaceutical Technology, Faculty of Pharmacy, University of Dhaka, Dhaka, Bangladesh.
}

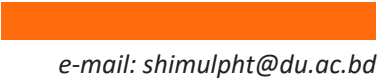

\section{ABSTRACT}

The present study aimed to compare the in vitro equivalence of different generic tablets of naproxen available with the reference brand in the Bangladesh pharmaceutical market. As naproxen belongs to the biopharmaceutics classification system (BCS) class-II drug, interchangeability among commercial products must be demonstrated through in vivo bioequivalence studies. However, in Bangladesh, such studies remain to be performed. An in vitro dissolution study was carried out using the United States Pharmacopoeia (USP) paddle method and the dissolution profiles of the generic products were compared with the reference brand. Quality assessment parameters including hardness, friability, and disintegration time were also evaluated. Similar dissolution behavior was found with the reference brand using a modeldependent and model-independent approach $\left(f_{2}>50, f_{1}<15\right)$, and there were no statistically significant differences. The best-fit kinetic model was the Korsmeyer-Peppas model, indicating that the mechanism of dissolution is predominantly diffusion. All commercial products complied with the official specifications. The results from this study suggest that the naproxen formulations available in the Bangladesh market could be used interchangeably for optimal therapeutic outcomes.

KEYWORDS: Naproxen, dissolution, similarity factor, difference factor, dissolution kinetics

\section{INTRODUCTION}

he oral solid dosage form is the most commonly used type of medicine, owing to ease of administration, high patient compliance, cost effectiveness, reduced sterility constraints, and flexibility of dosage form design (1). In spite of having tremendous advantages in oral administration, the major challenge with the design of oral dosage forms lies with the difficulty to control and maintain the quality in terms of oral absorption in the gastrointestinal (GI) tract due to its complex process of absorption (2). According to the World Health Organization (WHO), quality in the pharmaceutical industry is an important topic, especially concerning the assurance of delivering medicines to people with health needs in the developing world, where the chance of counterfeited medicines is a big challenge $(3,4)$. Dissolution studies of pharmaceutical solids has become one of the most important tools for analysis in drug product development and manufacturing, as well as in the regulatory assessment of pharmaceutical quality (5). The determination of drug dissolution in vitro is one of the key elements in the pharmaceutical development process and is sometimes used as a surrogate for the assessment of bioequivalence (6).

Naproxen is a highly potent non-steroidal antiinflammatory drug used to treat osteoarthritis, rheumatoid arthritis, and postoperative pain $(7,8)$. Consequently, naproxen has effective analgesic and anti-inflammatory properties with low gastrointestinal toxicity (9). Despite attractive pharmacological properties, naproxen has very poor aqueous solubility $(0.025 \mathrm{mg} / \mathrm{mL})$ and is a Biopharmaceutics Classification System (BCS) class II drug, whose bioavailability is ratelimited by its dissolution (10). In Bangladesh, around 50 generic products of naproxen are available. Among them, approximately seven products occupy the $90 \%$ share of naproxen. Due to the availability of many options, it is a big challenge for the regulatory authorities to assess and ensure the quality of products circulating in the market in order to expose substandard/counterfeit drugs (11).

The objectives of the present study were to assess the 
quality and dissolution profile of commercially available naproxen tablets with respect to the reference brand. The dissolution behavior was also treated analyzed using a model-dependent and model-independent approach to clarify the interchangeability of the commercially available naproxen tablets.

\section{MATERIALS AND METHODS}

\section{Materials}

Naproxen with a potency of $\geq 98 \%$ was generously supplied by Square Pharmaceuticals Ltd., Dhaka, Bangladesh. All other chemicals and reagents were of analytical/reagent grade and purchased from commercial sources.

\section{Collection of Samples}

Top-selling products of naproxen were selected based on data collected from local pharmacies. Samples were purchased from six different batches of every product from the model pharmacy and model medicine shops located in Dhaka city and coded randomly as NAP1-NAP6. Naprosyn (Roche) was used as the reference brand to compare with commercially available products in Bangladesh market.

The samples were properly checked for their physical appearance, name of the manufacturer, batch number, manufacturing date, expiry date, manufacturing license number, drug administration registration number, and maximum retail price at the time of purchase. Regarding sampling strategies, WHO guidelines for conducting a quality survey of medicines were followed (12). The experimental part of this work was undertaken at Stamford University Bangladesh.

\section{Determination of Naproxen}

The amount of naproxen in the commercial products and in reference brand were determined using a UV-visible spectrophotometer (UV-800, Shimadzu, Japan) at $262 \mathrm{~nm}$ according to a previous report (13). Potency was calculated against the standard solution as follows:

$\frac{\text { Absorbance of sample }}{\text { Absorbance of standard }} \times \frac{\text { concentration of standard solution }}{\text { concentration of sample solution }} \times$ potency of standard $(\%)$.

\section{Construction of Standard Curve}

A stock solution of naproxen $(1 \mathrm{mg} / \mathrm{mL})$ was prepared in ethanol. The stock solution was then diluted with dissolution media to prepare working solutions just before use (0.1-1.0 mg/mL). Naproxen concentration was analyzed by UV-spectrophotometer. The mean regression equation of standard calibration curves was $y=0.02162 x$ - 0.0117 (Fig. 1). Linear regression was significant $\left(R^{2}\right.$ $=0.9939 ; p=0.0001)$. The 95\% confidence interval $(\mathrm{Cl})$ estimated for the value of the intercept was -0.02032 to -0.003142 .

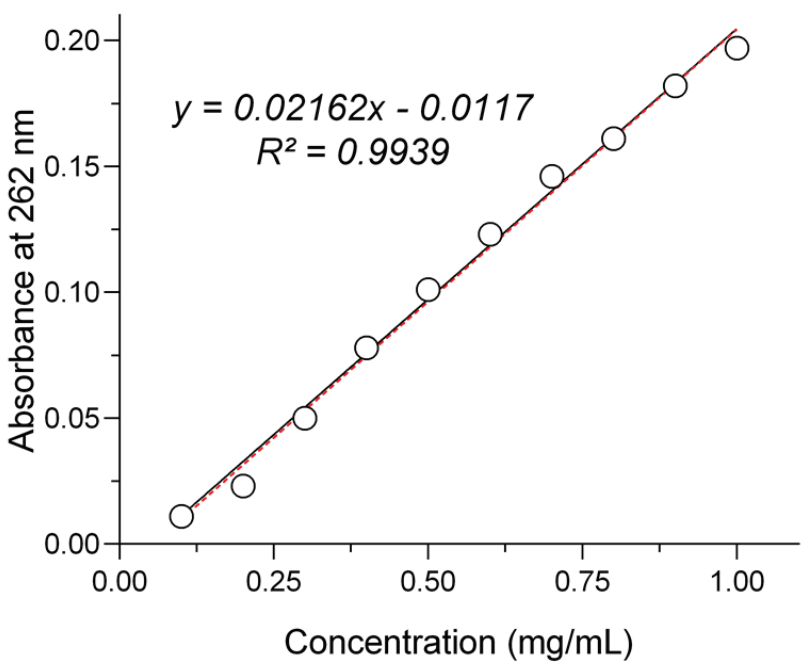

Figure 1. Calibration curve of naproxen reference standard.

\section{Preparation of Dissolution Media}

The dissolution media was simulated intestinal fluid (SIF) without enzymes prepared by mixing sodium dihydrogen phosphate and disodium hydrogen phosphate with dilution to achieve a final concentration $0.05 \mathrm{M}$ with a $\mathrm{pH}$ of 6.8 .

\section{Preparation of Samples}

Ten tablets of each commercial product of naproxen and the reference brand were weighed and finely powdered. A portion of powder equal to $10 \mathrm{mg}$ of naproxen was transferred to a $100-\mathrm{mL}$ volumetric flask and dissolved in $100 \mathrm{~mL}$ of dissolution media and filtered through 0.4- $\mu \mathrm{m}$ filter paper.

\section{Physical Evaluation of Naproxen Samples}

The tablet samples were physically evaluated (including thickness, diameter, hardness, friability, and weight variation) according to reported methods specified in the United States Pharmacopeia (14).

\section{In-vitro Disintegration Time}

The disintegration test was performed in distilled water using a USP disintegration apparatus at $37 \pm 0.5{ }^{\circ} \mathrm{C}$. The disintegration time was recorded when no particles persisted on the vessel, i.e., complete disintegration of naproxen samples was attained.

\section{Dissolution Test of Naproxen Samples}

Dissolution tests of naproxen samples were performed using a USP apparatus 2 (paddle) (Electrolab, EDT-08Lx, USA) for $60 \mathrm{~min}$ in $900 \mathrm{~mL}$ of SIF (pH 6.8) at $37 \pm 0.5$ ${ }^{\circ} \mathrm{C}(n=12$ tablets of each product). Samples $(0.5 \mathrm{~mL})$ were collected at $10,20,30,40,50$, and 60 minutes to measure the amount of naproxen. In brief, each sample was put into the dissolution vessel containing $900 \mathrm{~mL}$ of 
dissolution media and centrifuged at $10,000 \times \mathrm{g}$ for 5 min. The supernatant was diluted with an equal volume of dissolution media. The sampled dissolution media was not replaced.

\section{Model-Independent Fit Factors}

Fit factors are used to compare the dissolution profiles of test products with a reference product. The difference factor $\left(f_{1}\right)$ calculates the percentage difference between the two curves (reference and test drug) at each time point, whereas the similarity factor $\left(f_{2}\right)$ is a logarithmic reciprocal square root transformation of the sum of squared error and is a measurement of similarity in the dissolution percentage (3).

The parameter $f_{1}$, whose values range from 0 to 15 , and $f_{2}$, whose values range from 50 to 100 , ensure sameness or equivalence of the two dissolution curves for test and reference samples.

Another model-independent factor is mean dissolution time (MDT). MDT was determined from the accumulative curves of dissolved naproxen as a function of time (15).

In addition, we calculated dissolution efficiency (DE), which is the area under the dissolution curve within a time range (3).

\section{Model-Dependent Dissolution Kinetics}

To investigate the in vitro release kinetics and mechanism of drug release, various mathematical model-dependent kinetic models are used to describe drug dissolution from solid dosage forms (16). The kinetics of the dissolution process were studied through the analysis of dissolution data using several kinetic models, such as zero-order, first-order, Hixson-Crowell's cube root law, Higuchi's square root equation, Korsmeyer-Peppas, and Weibull's distribution. The best fitting equation uses coefficient of determination $\left(R^{2}\right)$, adjusted $R^{2}$, and Akaike information criterion (AIC) (17). The best-fit model is considered to have the lowest AIC and highest $R^{2}$ adjusted values (18).

\section{Statistical Analysis}

All data are represented as mean \pm standard deviation (SD) unless otherwise stated. The mathematical parameters were calculated using DDSolver (add-in for Microsoft Excel) (19). Graphs were created using GraphPad Prism 6.0 (GraphPad Software, LaJolla, CA).

\section{RESULTS AND DISCUSSION}

\section{Physical Evaluation of Naproxen Samples}

Based on results of physical evaluation (i.e., potency, thickness, diameter, hardness, friability, and weight variation), all samples complied with the USP guidelines, and there were no significant differences when test products were compared with the reference brand (Table 1) (14). From the results of the disintegration test (Table 1), it was found all samples complied with USP guidelines and disintegrated within 10 min except for samples 2 and 4 .

\section{In Vitro Dissolution Studies}

In commercial manufacturing of pharmaceuticals, the lotto-lot quality assurance is used to adopt the development of new formulations, secure homogeneity between lots or batches, and to confirm acceptable drug performance even after being modified (20). Among all in vitro quality parameters, the dissolution test is predominantly the best one to compare formulations, as dissolution is the rate and extent of drug substance that is transferred into solution from the solid matrix (21). The dissolution test helps in characterizing in vitro release rates and quality control, which can serve as a substitute for bioavailability and bioequivalence $(22,23)$.

Results of dissolution studies of naproxen samples are presented in Figure 2. All samples dissolved completely or almost completely within $60 \mathrm{~min}$. Per the USP

Table 1. Physical and Non-Compendial Parameters of Naproxen (NAP) Samples

\begin{tabular}{|c|c|c|c|c|c|c|c|}
\hline Sample & $\begin{array}{c}\text { Thickness }^{\mathrm{a}} \\
(\mathrm{mm})\end{array}$ & $\begin{array}{c}\text { Diameter }^{\mathrm{a}} \\
(\mathrm{mm})\end{array}$ & $\begin{array}{c}\text { Weight Variation } \\
(\%)\end{array}$ & $\begin{array}{c}\text { Friability }^{\mathrm{a}} \\
(\%)\end{array}$ & $\begin{array}{c}\text { Hardness }^{\mathrm{b}} \\
(\mathbf{k g} / \mathrm{cm} 2)\end{array}$ & $\begin{array}{c}\text { Disintegration } \\
\text { Time }^{\mathrm{b}} \\
(\mathrm{min})\end{array}$ & $\begin{array}{c}\text { Potency } \\
(\%)^{\mathrm{c}}\end{array}$ \\
\hline Reference & $5.97 \pm 0.02$ & $12.57 \pm 0.01$ & $1.01 \pm 0.39$ & 0.42 & $6.8 \pm 0.36$ & $1.74 \pm 0.56$ & $99.33 \pm 0.58$ \\
\hline NAP1 & $5.90 \pm 0.05$ & $10.83 \pm 0.05$ & $3.12 \pm 0.19$ & 0.13 & $8.1 \pm 0.56$ & $1.43 \pm 0.04$ & $99.39 \pm 0.81$ \\
\hline NAP2 & $6.46 \pm 0.11$ & $12.57 \pm 0.03$ & $2.26 \pm 0.46$ & 0.01 & $7.6 \pm 0.47$ & $12.6 \pm 0.57$ & $100.8 \pm 1.98$ \\
\hline NAP3 & $6.48 \pm 0.12$ & $13.83 \pm 0.06$ & $2.49 \pm 0.38$ & 0.02 & $8.0 \pm 0.43$ & $6.98 \pm 0.32$ & $96.68 \pm 0.56$ \\
\hline NAP4 & $7.07 \pm 0.04$ & $17.33 \pm 2.33$ & $3.18 \pm 0.25$ & 0.74 & $7.5 \pm 0.62$ & $11.7 \pm 0.59$ & $100.7 \pm 1.45$ \\
\hline NAP5 & $6.93 \pm 0.08$ & $10.82 \pm 0.03$ & $2.87 \pm 0.43$ & 0.06 & $6.6 \pm 0.63$ & $6.95 \pm 0.39$ & $96.24 \pm 0.57$ \\
\hline NAP6 & $17.2 \pm 0.10$ & $17.35 \pm 0.79$ & $1.54 \pm 0.57$ & 0.10 & $7.1 \pm 0.43$ & $5.38 \pm 0.19$ & $96.94 \pm 0.59$ \\
\hline
\end{tabular}

Data are expressed as mean $\pm S D$.

${ }^{a} n=10 ;{ }^{b} n=6 ;{ }^{c} n=3$. 
specification, naproxen must release at least $80 \%$ within 45 min using USP apparatus type II (24). In the present study, all commercial products and the reference brand satisfied the USP specification (Table 2).

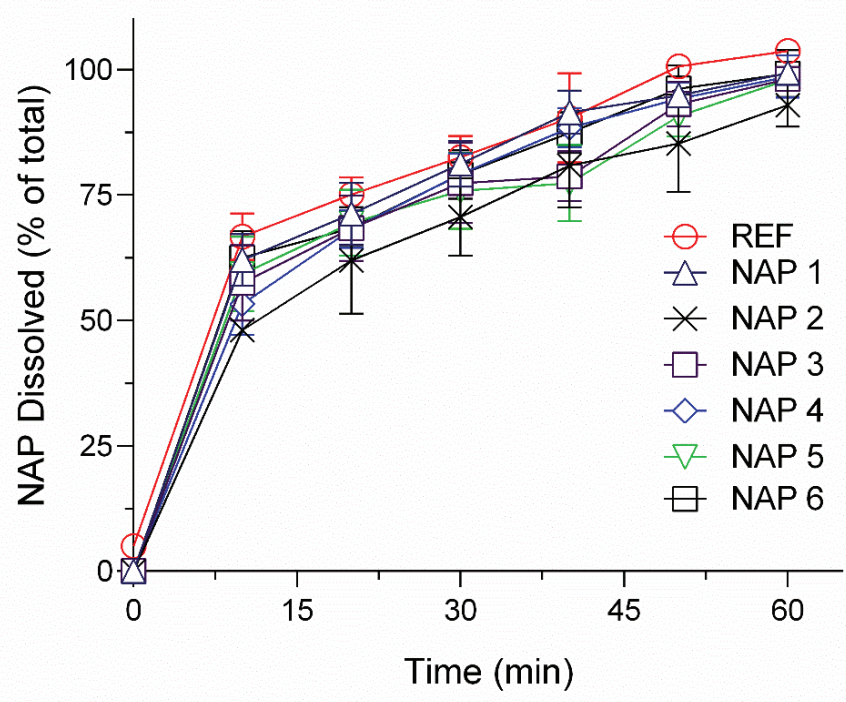

Figure 2. Comparative in vitro dissolution profiles of naproxen (NAP) samples at $p H$ 6.8. Data are expressed as mean \pm SD. Ref: Reference.

Table 2. Various Dissolution Related Model-Independent Fit Factors of Naproxen (NAP) Samples

\begin{tabular}{|c|c|c|c|c|c|c|}
\hline Sample & $f_{2}$ & $f_{1}$ & $\begin{array}{c}\mathrm{MDT} \\
(\mathrm{min})\end{array}$ & $\begin{array}{c}\mathrm{T}_{50} \\
(\mathrm{~min})\end{array}$ & $\begin{array}{c}\mathrm{T}_{80} \\
(\mathrm{~min})\end{array}$ & $\begin{array}{c}\mathrm{DE} \\
(\%)\end{array}$ \\
\hline Reference & & & 14.32 & 5.01 & 29.45 & 73.3 \\
\hline NAP1 & 76.6 & 2.59 & 13.18 & 5.13 & 27.15 & 75.1 \\
\hline NAP2 & 54.4 & 10.08 & 19.93 & 11.32 & 40.38 & 65.6 \\
\hline NAP3 & 73.7 & 3.25 & 16.09 & 6.70 & 33.16 & 70.7 \\
\hline NAP4 & 71.7 & 3.38 & 15.47 & 8.05 & 31.60 & 72.1 \\
\hline NAP5 & 70.6 & 3.76 & 16.50 & 5.94 & 33.96 & 70.3 \\
\hline NAP6 & 90.0 & 1.46 & 13.72 & 4.90 & 28.22 & 73.9 \\
\hline
\end{tabular}

Data are expressed as mean values.

$f_{2}$, Similarity factor; $f_{1}$, difference factor; $M D T$, mean dissolution time; $T_{50}$, time to dissolve $50 \%$ of NAP; $T_{80}$, time to dissolve $80 \%$ of NAP; and DE, dissolution efficiency.

\section{Dissolution Profile Comparison}

Dissolution profiles of naproxen samples including the reference brand were subjected to non-linear regression analysis by fitting both model-dependent and modelindependent factors. According to the U.S. Food and Drug Administration (FDA), fit factors are important quantitative parameters used to explain and compare the dissolution profiles of different samples with the reference brand. As per FDA guidelines, $f_{2}$ is more accurate in identifying the dissimilarities among samples; an $f_{2}$ value greater than 50 indicates identical dissolution behavior. On the other hand, $f_{1}$ clarifies the difference in the dissolution profile based on sampling times. The $f_{1}$ value must be in the range of $0-15$ to be considered identical dissolution behavior (25). Additional modelindependent parameters used to assess similarity in the present study were DE and MDT. The test products are considered to be similar when their DE and MDT values are close to that of reference product (within $\pm 10 \%$ is often acceptable) (25).

Results of model-independent analysis are presented in Table 2. Based on $f_{1}, f_{2}$, DE, and MDT values, all samples were considered to be similar to the reference product. This implies that the top-selling naproxen samples can be considered as interchangeable with the reference brand in Bangladesh.

In addition, several model-dependent kinetic models were evaluated by fitting experimental data of the reference and other products to explain the overall release of naproxen from samples. The model that gives a high $R^{2}$ value is considered as the best-fitted model of the release data (26). In Table 3, the $R^{2}$, adjusted $R^{2}$, dissolution coefficient $(n)$, and AIC values are presented to identify and clarify the best-fit model. The KorsmeyerPeppas model provides the highest $R^{2}$ and adjusted $R^{2}$ and the lowest AIC values. This model is generally used to analyze the release of pharmaceutical exponents, which is indicated for polymeric dosage forms when the release mechanism is not well known or when more than one type of release phenomena is involved (27). The Korsmeyer-Peppas model also resulted in an $\mathrm{n}$ value lower than 0.45 for all formulations, indicating that the mechanism of dissolution is predominantly diffusion (Fickian case - I) (17). From the results of kinetic analysis, it can be declared that all naproxen samples including the reference brand in this study exhibited a similar release mechanism.

\section{CONCLUSION}

This study attempted to compare the quality and dissolution behavior of generic naproxen tablets with the reference brand available in Bangladesh. The quality evaluation showed that all naproxen samples met the USP specifications. The dissolution evaluation showed that all naproxen samples were similar to the reference brand. Therefore, naproxen tablets available in the Bangladesh pharmaceutical market have similar biopharmaceutical behaviors as the reference brand and may be used interchangeably.

\section{ACKNOWLEDGEMENT}

The authors thank Square Pharmaceuticals Ltd., Dhaka, 


\begin{tabular}{|c|c|c|c|c|c|c|c|c|}
\hline \multirow{2}{*}{ Model } & \multirow{2}{*}{ Parameters } & \multicolumn{7}{|c|}{ Samples } \\
\hline & & Reference & NAP1 & NAP2 & NAP3 & NAP4 & NAP5 & NAP6 \\
\hline \multirow{4}{*}{ Zero Order } & $R^{2}$ & 0.7620 & 0.7499 & 0.8293 & 0.7797 & 0.8077 & 0.7591 & 0.7651 \\
\hline & Adjusted $R^{2}$ & 0.7144 & 0.6999 & 0.7952 & 0.7356 & 0.7692 & 0.7109 & 0.7181 \\
\hline & $\mathrm{K}_{0}$ & 2.029 & 2.071 & 1.859 & 1.973 & 2.026 & 1.953 & 2.047 \\
\hline & AIC & 50.87 & 51.35 & 48.05 & 50.10 & 49.68 & 50.45 & 50.90 \\
\hline \multirow{4}{*}{ First-Order } & $R^{2}$ & 0.8897 & 0.8891 & 0.9680 & 0.8557 & 0.9347 & 0.8084 & 0.8875 \\
\hline & Adjusted $R^{2}$ & 0.8621 & 0.8613 & 0.9600 & 0.8197 & 0.9184 & 0.7605 & 0.8593 \\
\hline & $K_{1}$ & 0.065 & 0.071 & 0.046 & 0.058 & 0.060 & 0.058 & 0.066 \\
\hline & AIC & 36.61 & 33.99 & 33.17 & 37.00 & 29.43 & 38.74 & 36.35 \\
\hline \multirow{4}{*}{ Higuchi } & $R^{2}$ & 0.9843 & 0.9877 & 0.9964 & 0.9656 & 0.9918 & 0.9480 & 0.9803 \\
\hline & Adjusted $R^{2}$ & 0.9804 & 0.9846 & 0.9955 & 0.9571 & 0.9898 & 0.9350 & 0.9754 \\
\hline & $\mathrm{K}_{\mathrm{h}}$ & 13.88 & 14.18 & 12.62 & 13.46 & 13.79 & 13.35 & 13.99 \\
\hline & AIC & 38.95 & 39.45 & 31.64 & 37.73 & 34.60 & 39.11 & 38.88 \\
\hline \multirow{4}{*}{ Hixon-Crowell } & $R^{2}$ & 0.9540 & 0.9767 & 0.9904 & 0.9242 & 0.9923 & 0.8900 & 0.9614 \\
\hline & Adjusted $R^{2}$ & 0.9424 & 0.9709 & 0.9879 & 0.9053 & 0.9904 & 0.8625 & 0.9518 \\
\hline & $K_{d}$ & 0.017 & 0.018 & 0.012 & 0.015 & 0.016 & 0.015 & 0.017 \\
\hline & AIC & 40.55 & 38.62 & 38.34 & 40.89 & 35.74 & 42.28 & 40.16 \\
\hline \multirow{5}{*}{$\begin{array}{c}\text { Korsmeyer- } \\
\text { Peppas }\end{array}$} & $R^{2}$ & 0.9739 & 0.9864 & 0.9971 & 0.9665 & 0.9946 & 0.9447 & 0.9629 \\
\hline & Adjusted $R^{2}$ & 0.9674 & 0.9830 & 0.9963 & 0.9582 & 0.9933 & 0.9309 & 0.9537 \\
\hline & $K_{k p}$ & 30.59 & 31.94 & 20.61 & 27.51 & 24.37 & 29.71 & 30.37 \\
\hline & $\mathbf{n}$ & 0.284 & 0.278 & 0.366 & 0.305 & 0.345 & 0.281 & 0.288 \\
\hline & AIC & 24.40 & 19.86 & 12.71 & 27.58 & 13.46 & 29.42 & 25.38 \\
\hline \multirow{4}{*}{ Weibul } & $R^{2}$ & 0.8963 & 0.9354 & 0.9749 & 0.8944 & 0.9740 & 0.8622 & 0.8886 \\
\hline & Adjusted $R^{2}$ & 0.8704 & 0.9193 & 0.9687 & 0.8680 & 0.9674 & 0.8278 & 0.8608 \\
\hline & $\beta$ & 0.638 & 0.686 & 0.715 & 0.644 & 0.802 & 0.581 & 0.658 \\
\hline & AIC & 32.14 & 29.35 & 25.16 & 32.76 & 25.88 & 33.50 & 32.85 \\
\hline
\end{tabular}

$R^{2}$, correlation coefficient; adjusted $R^{2}$, adjusted correlation coefficient using nonlinear regression; $k_{0}$, zero-order release constant; $k_{1}$, first-order release constant; $K_{h}$, Higuchi rate constant; $K_{d}$, Hixson-Crowell kinetics constant; $K_{k p}$, Korsmeyer release rate constant; $n$, diffusion coefficient; 6 , shape parameter; AIC, Akaike Information Criteria.

Bangladesh for generously providing the naproxen reference drug.

\section{CONFLICT OF INTEREST}

The authors disclosed no conflicts of interest related to this article.

\section{REFERENCES}

1. Baghel, S.; Cathcart, H.; O'Reilly, N. J. Polymeric Amorphous Solid Dispersions: A Review of Amorphization, Crystallization, Stabilization, Solid-State Characterization, and Aqueous Solubilization of Biopharmaceutical Classification System Class II Drugs. J. Pharm. Sci. 2016, 105, 2527-2544. DOI: 10.1016/j.xphs.2015.10.008.

2. Kalepu, S.; Nekkanti, V. Insoluble drug delivery strategies: review of recent advances and business prospects. Acta Pharm. Sin. B 2015, 5, 442-453. DOI: 10.1016/j. apsb.2015.07.003.
3. Hambisa, S.; Belew, S.; Suleman, S. In vitro comparative quality assessment of different brands of norfloxacin tablets available in Jimma, SouthWest Ethiopia. Drug Des. Devel. Ther. 2019, 13, 1241-1249. DOI: 10.2147/DDDT. S189524.

4. WHO Expert Committee on Specifications for Pharmaceutical Preparations. Quality Assurance of Pharmaceuticals; WHO Technical Report Series, No. 902, World Health Organization: Geneva, 2007, pp 17-57.

5. Lee, S. L.; Raw, A. S.; Yu, L. Dissolution Testing. In Biopharmaceutics Applications in Drug Development; Krishna, R.; Yu, L.; Eds. Springer: Boston, 2008. DOI: 10.1007/978-0-387-72379-2_3.

6. Agata, Y.; Iwao, Y.; Miyagishima, A.; Itai, S. Novel mathematical model for predicting the dissolution profile of spherical particles under non-sink conditions. Chem. Pharm. Bull. 2010, 58, 511-515. DOI: 10.1248/cpb.58.511. 
7. Ho, K. Y.; Gwee, K. A.; Cheng, Y. K.; Yoon, K. H.; Hee, H. T.; Omar, A. R. Nonsteroidal anti-inflammatory drugs in chronic pain: implications of new data for clinical practice. J. Pain Res. 2018, 11, 1937-1948. DOI: 10.2147/JPR. S168188.

8. Couto, A.; Troullos, E.; Moon, J.; Paredes-Diaz, A.; An, R. Analgesic efficacy and safety of non-prescription doses of naproxen sodium in the management of moderate osteoarthritis of the knee or hip. Curr. Med. Res. Opin. 2018, 34, 1747-1753. DOI: 10.1080/03007995.2018.1437029.

9. Brogden, R. N.; Heel, R. C.; Speight, T. M.; Avery, G. S. Naproxen up to date: a review of its pharmacological properties and therapeutic efficacy and use in rheumatic diseases and pain states. Drugs 1979, 18, 241-277. DOI: 10.2165/00003495-197918040-00001.

10. Tsume, Y.; Mudie, D. M.; Langguth, P.; Amidon, G. E.; Amidon, G. L. The Biopharmaceutics Classification System: subclasses for in vivo predictive dissolution (IPD) methodology and IVIVC. Eur. J. Pharm. Sci. 2014, 57, 152163. DOI: 10.1016/j.ejps.2014.01.009.

11. Glass, B. D. Counterfeit drugs and medical devices in developing countries. Res. Rep. Trop. Med. 2014, 5, 11-22. DOI: 10.2147/RRTM.S39354.

12. Guidelines on the Conduct of Surveys of the Quality of Medicines; Working Document QAS/15.630; World Health Organization; Geneva, June 2015.

13. Jain, N. A.; Lohiya, R. T.; Umekar M. J. Spectrophotometric determination of naproxen and esomeprazole in a laboratory mixture by simultaneous equation, absorption correction, absorption ratio and area under curve methods. IJPSR. 2011, 2, 130-134.

14. The United States Pharmacopeia and National Formulary USP 41-NF 36; The United States Pharmacopeial Convention, Inc.: Rockville, MD, 2018.

15. Medina, J. R.; Salazar, D. K.; Hurtado, M.; Cortés, A. R.; Domínguez-Ramírez, A. M. Comparative in vitro dissolution study of carbamazepine immediate-release products using the USP paddles method and the flow-through cell system. Saudi Pharm. J. 2014, 22, 141-147. DOI: 10.1016/j. jsps.2013.02.001.

16. Siepmann, J.; Peppas, N. A. Modeling of drug release from delivery systems based on hydroxypropyl methylcellulose (HPMC). Adv. Drug Deliv. Rev. 2012, 64 (suppl), 163-174. DOI: 10.1016/j.addr.2012.09.028.

17. Mathematical models of drug release. In Strategies to Modify Drug Release from Pharmaceutical Systems;
Bruschi, M. L.; Ed. Woodhead Publishing, 2015, pp 63-86. DOI: 10.1016/B978-0-08-100092-2.00005-9.

18. Parezanović, G. Š.; Lalić-Popović, M.; Goločorbin-Kon, S.; Todorović, N.; Pavlović, N.; Mikov, M. In vitro comparative quality evaluation of non-expired and 10 years-expired lamotrigine immediate-release tablet formulations - pilot study. Dissolut. Technol. 2020, 27, 14-20. DOI: 10.14227/ DT270120P14.

19. Zhang, Y.; Huo, M.; Zhou, J.; Zou, A.; Li, W.; Yao, C.; Xie, S. DDSolver: An add-in program for modeling and comparison of drug dissolution profiles. AAPS J. 2010, 12, 263-271. DOI: 10.1208/s12248-010-9185-1.

20. Shah, V. P. Progressive applications of dissolution, its impact, and implications in the pharmaceutical world. J. Pharm. Sci. 2013, 102, 2895-2897. DOI: 10.1002/jps.23608.

21. Kawabata, Y.; Wada, K.; Nakatani, M.; Yamada, S.; Onoue, S. Formulation design for poorly water-soluble drugs based on Biopharmaceutics Classification System: basic approaches and practical applications. Int. J. Pharm. 2011, 420, 1-10. DOI: 10.1016/j.ijpharm.2011.08.032.

22. Anand, O.; Yu, L. X.; Conner, D. P.; Davit, B. M. Dissolution testing for generic drugs: an FDA perspective. AAPS J. 2011, 13, 328-335. DOI: 10.1208/s12248-011-9272-y.

23. Dickinson, P. A.; Lee, W. W.; Stott, P. W.; Townsend, A. I.; Smart, J. P.; Ghahramani, P.; Hammett, T.; Billett, L.; Behn, S.; Gibb, R. C.; Abrahamsson, B. Clinical relevance of dissolution testing in quality by design. AAPS J. 2008, 10, 380-390. DOI: 10.1208/s12248-008-9034-7.

24. Naproxen Sodium Tablets. In The United States Pharmacopeia and National Formulary. USP 41-NF 36; The United States Pharmacopeial Convention, Inc.: Rockville, MD, 2018, pp 5-6.

25. Anderson, N. H.; Bauer, M.; Boussac, N.; Khan-Malek, R.; Munden, P.; Sardaro, M. An evaluation of fit factors and dissolution efficiency for the comparison of in vitro dissolution profiles. J. Pharm. Biomed. Anal. 1998, 17, 811822. DOI: 10.1016/S0731-7085(98)00011-9.

26. Mircioiu, C.; Voicu, V.; Anuta, V.; Tudose, A.; Celia, C.; Paolino, D.; Fresta, M.; Sandulovici, R.; Mircioiu, I. Mathematical modeling of release kinetics from supramolecular drug delivery systems. Pharmaceutics 2019, 11, 1-45. DOI: 10.3390/pharmaceutics11030140.

27. Mohapatra, S.; Kar, R. K.; Sahoo, S. K. Goodness of fit model dependent approaches of controlled release matrix tablets of zidovudine. Indian J. Pharm. Educ. Res. 2016, 50, 138-145. DOI: 10.5530/ijper.50.1.18. 